\title{
Neurofeedback Training versus Treatment-as- Usual for Alcohol Dependence: Results of an Early-Phase Randomized Controlled Trial and Neuroimaging Correlates
}

\author{
Leena Subramanian $^{a}$ Leon Skottnik ${ }^{b}$ W. Miles Cox ${ }^{c}$ Michael Lührs ${ }^{d, e}$ \\ Rachel McNamara $^{f}$ Kerry Hood ${ }^{f}$ Gareth Watson $^{f}$ Joseph R. Whittakerg \\ Angharad N. Williams $^{\text {h }}$ Raman Sakhuja ${ }^{i}$ Niklas Ihssen $^{j}$ Rainer Goebeld, e \\ Rebecca Playle $^{f}$ David E.J. Linden ${ }^{a, b}$

\begin{abstract}
aMR Centre for Neuropsychiatric Genetics and Genomics and Cardiff University Brain Research Imaging Centre,

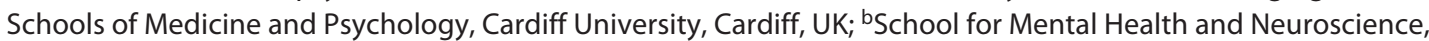
Faculty of Health, Medicine and Life Sciences, Maastricht University, Maastricht, The Netherlands; 'School of Psychology, Bangor University, Bangor, UK; ${ }^{d}$ Department of Cognitive Neuroscience, Faculty of Psychology

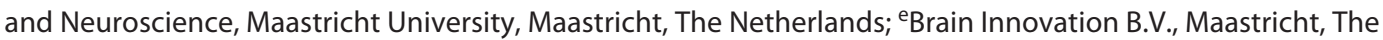

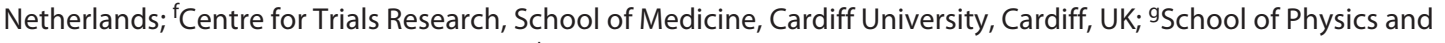
Astronomy, Cardiff University, Cardiff, UK; hAdaptive Memory Research Group, Max Planck Institute for Human Cognitive and Brain Sciences, Leipzig, Germany; 'Addiction Services, Cwm Taf Morgannwg University Health Board, Mountain Ash, UK; 'Department of Psychology, Durham University, Durham, UK
\end{abstract}

\section{Keywords}

Addiction · Alcohol dependence · Neurofeedback ·

Real-time functional magnetic resonance imaging •

Self-regulation

\begin{abstract}
Introduction: Alcohol dependence is one of the most common substance use disorders, and novel treatment options are urgently needed. Neurofeedback training (NFT) based on real-time functional magnetic resonance imaging (rtfMRI) has emerged as an attractive candidate for add-on treatments in psychiatry, but its use in alcohol dependence has not been formally investigated in a clinical trial. We investigated the use of rtfMRI-based NFT to prevent relapse in alcohol dependence. Methods: Fifty-two alcohol-depen-
\end{abstract}

karger@karger.com www.karger.com/ear

Karger $\stackrel{\text { ' }}{5}$

GOPEN ACCESS
(C) 2021 The Author(s)

Published by S. Karger AG, Basel

This is an Open Access article licensed under the Creative Commons Attribution-NonCommercial-4.0 International License (CC BY-NC) (http://www.karger.com/Services/OpenAccessLicense), applicable to the online version of the article only. Usage and distribution for commercial purposes requires written permission. dent patients from the UK who had completed a detoxification program were randomly assigned to a treatment group (receiving rtfMRI NFT in addition to standard care) or the control group (receiving standard care only). At baseline, alcohol consumption was assessed as the primary outcome measure and a variety of psychological, behavioral, and neural parameters as secondary outcome measures to determine feasibility and secondary training effects. Participants in the treatment group underwent 6 NFT sessions over 4 months and were trained to downregulate their brain activation in the salience network in the presence of alcohol stimuli and to upregulate frontal activation in response to pictures related to positive goals. Four, 8, and 12 months after baseline assessment, both groups were followed up with a

Leena Subramanian and Leon Skottnik contributed equally.
Leon Skottnik

School for Mental Health and Neuroscience, Maastricht University Vijverdalseweg 1

NL-62226 Maastricht (The Netherlands)

l.skottnik@maastrichtuniversity.nl 
battery of clinical and psychometric tests. Results: Primary outcome measures showed very low relapse rates for both groups. Analysis of neural secondary outcome measures indicated that the majority of patients modulated the salience system in the desired directions, by decreasing activity in response to alcohol stimuli and increasing activation in response to positive goals. The intervention had a good safety and acceptability profile. Conclusion: We demonstrated that rtfMRI-neurofeedback targeting hyperactivity of the salience network in response to alcohol cues is feasible in currently abstinent patients with alcohol dependence.

(C) 2021 The Author(s)

Published by S. Karger AG, Basel

\section{Introduction}

Neurofeedback, a type of biofeedback that utilizes brain signals for self-regulation training, has seen a recent rise in clinical interest because of the development of realtime functional magnetic resonance imaging (rtfMRI). For neurofeedback training (NFT), rtfMRI offers benefits when compared to EEG neurofeedback. For example, fMRI can be used to localize brain signals in specific regions of the brain with greater precision, and patients can activate targeted brain areas by using specific mental imagery (e.g., imagining moving a body part). In pilot investigations, rtfMRI NFT has already been tested for its potential to help patients with various kinds of substance use disorder to attenuate their symptoms, reduce the frequency or intensity of their unwanted behavior, and/or modulate putative disease-related brain activity [1-5].

We conducted an early-phase randomized controlled trial for alcohol dependence, in which we compared NFT plus treatment-as-usual (TAU) versus TAU only [6]. Participants in the neurofeedback group were exposed to 2 categories of stimuli while they were in the scanner. One category comprised alcohol-related pictures, such as images of alcoholic drinks. The other category comprised pictures that were related to socially desirable and healthy goals (those related to, e.g., employment, family, or personal relationships) whose pursuit might serve as an alternative to drinking alcohol. Participants received feedback about the degree of activation in specific brain regions that the stimuli in each category activated, and they were trained to regulate these responses - either to downregulate their responses to the alcohol-related stimuli or to upregulate their responses to the alternative goal stimuli. The inclusion of both alcohol-related and alternative goal-related stimuli was consistent with motivational the- ory [7-9], which has shown that problematic drinkers are better able to regulate their drinking if they have healthy, alternative goals to pursue. For testing this tenet of motivational theory, we had also previously shown that at a neural level, heavy drinkers overvalue alcohol and undervalue other goals that they might pursue and enjoy as an alternative to drinking alcohol [10]. The objectives of this early-phase randomized controlled trial were to determine (a) the feasibility of the approach, that is, whether participants who received rtfMRI NFT would be able to downregulate their brain activation in the presence of the alcohol-related stimuli and to upregulate their brain activation in the presence of the alternative goal-related stimuli and (b) whether the training would be associated with changes on our primary outcome measures, that is, reductions in alcohol consumption, reductions in urges to drink, and with improvements in other areas of functioning (efficacy).

\section{Materials and Methods}

\section{Patients, Eligibility, and Randomization}

Fifty-two participants with alcohol dependence were recruited from 2015 to 2017 through NHS alcohol services in southeast Wales (Cardiff and Vale University Health Board, Cwm Taf University Health Board, and Aneurin Bevan University Health Board) with the help of collaborating clinicians specializing in addiction, staff from the Health and Care Research Wales Clinical Research Centre and through alcohol addiction rehabilitation centers.

Participants were eligible to take part in the study if they were older than 18 years, had a clinical diagnosis of alcohol dependence, were within 1-6 months of having successfully completed an inpatient detoxification program, and had been abstinent from alcohol use since detoxification and at the time of recruitment. The exclusion criteria were ongoing regular abuse of illicit substances (except for cannabis), a history of a psychotic disorder not related to alcohol, an IQ $<70$, involvement in other interventional research within the 6 months prior to recruitment, and non-fulfillment of the MRI safety requirements.

Randomization was carried out through an online system overseen by the Centre for Trials Research, Cardiff University. Participants were randomized into either the neurofeedback treatment (NFT) group or the TAU group. The randomization method used time since detoxification (1-3 months and 4-6 months) as a minimization factor.

Standard Protocol Approval, Registration, and Patient Consent

This study was approved by the Wales Research Ethics Committee 1 (Ref: 14/WA/1172), the Cardiff School of Psychology Ethics Committee, and the Cwm Taf, Cardiff and Vale University, and Aneurin Bevan University Health Boards. All participants gave written informed consent to participate in the study in accordance with the Declaration of Helsinki. The trial was registered with Clinicaltrials.gov (NCT02486900) in June 2015 before the first 
participant was recruited. The study was completed in August 2018 after acquisition of the final follow-up assessments.

\section{Design}

Fifty-two patients were randomly allocated (parallel assignment) to receive either the neurofeedback intervention along with TAU NFT group or TAU control group. TAU included psychoeducation, psychological support, and medical management of abstinence with, for example, the use of disulfiram or anti-craving medication. After completing the study, patients in the control group were offered 1 free NFT session. The NFT group received six 1-h sessions of NFT. The study was completed in August 2018 after acquisition of the final follow-up assessments. Figure 1 shows a flowchart of the study procedure.

\section{Psychological and Behavioral Outcome Measures}

At all 4 time points, the clinical outcome measure of alcohol consumption, the Alcohol Timeline Followback (TLFB [11]), was assessed and constituted the primary outcome measure at the 4-month time point and a secondary outcome measure at subsequent time points. All other secondary outcome measures were also acquired at all 4 time points, that is, for craving-related constructs, the Drinking Urge Questionnaire (DUQ [12]), the Obsessive Compulsive Drinking Scale (OCDS [13]), and alcohol Stroop test [14], and for emotion/mood-related processes, the Profile of Mood States (POMS [15]), the Hospital Anxiety and Depression Scale (HADS) [16], and Beck Depression Inventory (BDI-II [17]). Additionally, in the NFT group, alcohol craving was measured during the MRI session as a safety measure, that is, to track whether the images would induce craving. Ratings on a 5-point self-rating scale (0: no desire to drink alcohol; 4: very strong desire) were acquired for each of the neurofeedback/mirror runs in each session. Furthermore, participants self-rated their craving before and after each neurofeedback session by using the DUQ [12]. (For screening and overall baseline assessments, see [12].)

\section{NFT Protocol for the NFT Group}

During the baseline assessment, participants rated the alcoholrelated pictures, in relation to how much each picture made them want to drink alcohol. For the pictures related to alternative goals, participants indicated how much each one reminded them of a positive goal that they were currently pursuing. Based on these ratings, individual images were selected for the localizer task (see [6] for details). Patients in the NFT group subsequently received six 1 -h sessions of NFT in the MRI scanner (see [6]). In short, the first run of each session served as the localizer procedure in which alternating blocks of neutral pictures and either alcohol-related pictures or alternative goal-related pictures, were presented to the participants. Brain clusters activated during presentation of the alcohol/goal-related pictures in comparison to those activated by the neutral images were used to create individual neurofeedback regions for the subsequent neurofeedback runs (SI). The 4 scanning blocks (runs) that followed consisted of 2 neurofeedback and 2 mirror runs in alternating order. Neurofeedback was provided by scaling the size of the pictures presented according to the ROI signal (see fMRI data acquisition and feedback generation), which participants attempted to decrease while seeing the alcohol pictures and to increase while seeing the pictures related to alternative goals. During the mirror runs, participants passively viewed the stimuli, and the size of the image changed in accordance with the previous neurofeedback run and did not contain any information about their current level of brain activation.

During alcohol runs (neurofeedback and mirror runs), participants were instructed to downregulate their brain activity (using mental imagery) in the alcohol-related brain areas by decreasing the size of the alcohol-related images. Participants were instructed to think of ways to reduce the size of the images, and they were given continuous feedback about their brain activity in the target region. During the alternative goal-related runs, participants were instructed to upregulate brain activity in the target region (chosen for each individual while they viewed pictures related to positive personal goals).

After each session, participants were debriefed about their experience and given the opportunity to raise any concerns about their craving or other adverse effects of the procedure for which counseling would have been available. This however was never required. Once participants had identified their individual strategies (i.e., the strategy that they subjectively judged as being most suited) for decreasing the size of the alcohol-related pictures or for increasing the size of the pictures related to their alternative goals, they were asked as homework to spend 10 min 2-3 times a week using the imagining strategies that worked best.

\section{fMRI Data Acquisition and Feedback Generation}

The Siemens and GE MRI scanners at the Cardiff University Brain Research Imaging Centre were used to acquire the functional and anatomical scans. A high-resolution T1-weighted anatomical scan (24 slices) was acquired. Functional data were obtained using a standard BOLD EPI sequence (TR $=1,500 \mathrm{~ms}$; $\mathrm{TE}=30 \mathrm{~ms}$, 24 slices; $2.5 \mathrm{~mm}$ thick; $0.5 \mathrm{~mm}$ gap; 80 flip angle). A total of 300 volumes were acquired in each functional run for each participant. Acquired EPI data were transferred to a separate computer, which in real-time ran Turbo-BrainVoyager (version 3.2, Brain Innovation, Maastricht, The Netherlands) and was used to perform real-time trilinear motion correction and spatial smoothing (FWHM $4 \mathrm{~mm}$ ) and linear trend removal. For the localizer scan, real-time statistical analyses were carried out via an incremental general linear model (GLM). All further real-time analyses were performed using Turbo-BrainVoyager. Based on the computed feedback signal, the size of presented images was scaled (see [6] for details) using StimulGL. To control for physiological confounding factors of the BOLD signal (offline), physiological signals were acquired with Spike2 (version 5.21, Cambridge Electronics Design Ltd., Cambridge, UK). The heart rate was also recorded using photoplethysmography, the respiratory rate with a chest belt, and partial pressure of end-tidal carbon dioxide $\left(\mathrm{P}_{\mathrm{ET}} \mathrm{CO}_{2}\right)$ via a nasal cannula.

\section{Sample Size Calculation}

There were no published randomized trials to inform a sample size calculation for the early-phase efficacy trial of the neurofeedback intervention that was being evaluated. However, experimental neurofeedback studies have found large effect sizes of $0.5-1.5$, depending on the patient group that was tested, for example [2]. Thus, in the prespecified power analysis [6], we expected that a final sample size of 40 (20 in each group) would allow us to detect effect sizes ranging from 0.8 with $70 \%$ power to 1.1 with $90 \%$ power at a significance level of 0.05 . This is appropriate for single-site, early-phase detection of clinically promising results. 


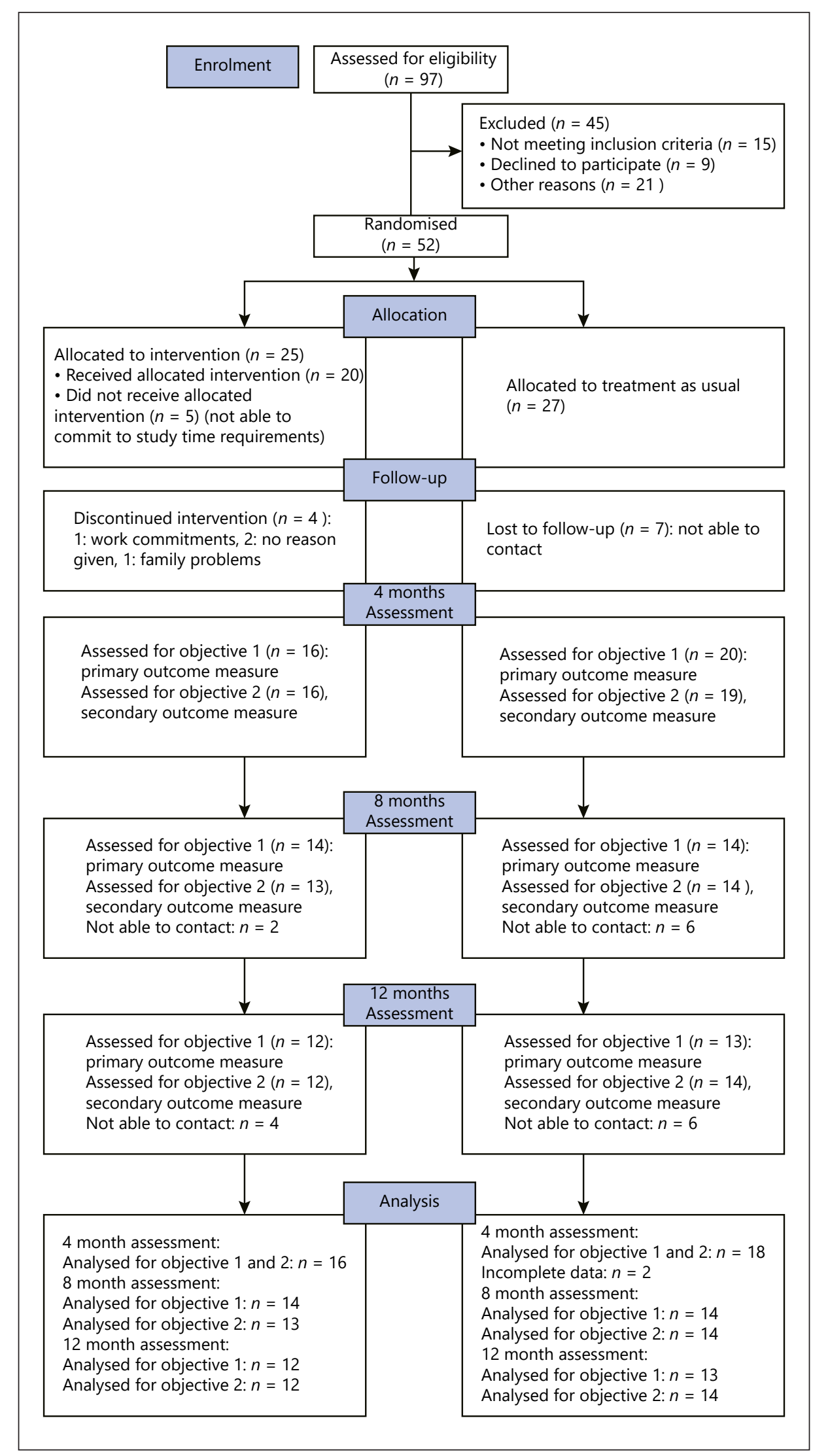

Fig. 1. CONSORT flow diagram of the phases of the trial. TAU, treatment-asusual; CONSORT, Consolidated Standards of Reporting Trials.
- Did not receive allocated

intervention $(n=5)$ (not able to commit to study time requirements)

Discontinued intervention ( $n=4$ )

1: work commitments, 2: no reason given, 1 : family problems

Assessed for objective $1(n=16)$ primary outcome measure Assessed for objective $2(n=16)$ secondary outcome measure

Assessed for objective $2(n=19)$, secondary outcome measure

\begin{tabular}{|c|c|c|}
\hline$\downarrow$ & 8 months & $\downarrow$ \\
\hline $\begin{array}{l}\text { Assessed for objective } 1(n=14) \text { : } \\
\text { primary outcome measure } \\
\text { Assessed for objective } 2(n=13) \text {, } \\
\text { secondary outcome measure } \\
\text { Not able to contact: } n=2\end{array}$ & & $\begin{array}{l}\text { Assessed for objective } 1(n=14) \text { : } \\
\text { primary outcome measure } \\
\text { Assessed for objective } 2(n=14) \text {, } \\
\text { secondary outcome measure } \\
\text { Not able to contact: } n=6\end{array}$ \\
\hline$\downarrow$ & $\begin{array}{l}12 \text { months } \\
\text { Assessment }\end{array}$ & $\downarrow$ \\
\hline $\begin{array}{l}\text { Assessed for objective } 1(n=12) \text { : } \\
\text { primary outcome measure } \\
\text { Assessed for objective } 2(n=12) \text {, } \\
\text { secondary outcome measure } \\
\text { Not able to contact: } n=4\end{array}$ & & $\begin{array}{l}\text { Assessed for objective } 1(n=13) \text { : } \\
\text { primary outcome measure } \\
\text { Assessed for objective } 2(n=14) \text {, } \\
\text { secondary outcome measure } \\
\text { Not able to contact: } n=6\end{array}$ \\
\hline$\downarrow$ & Analysis & $\downarrow$ \\
\hline $\begin{array}{l}4 \text { month assessment: } \\
\text { Analysed for objective } 1 \text { and 2: } n=16 \\
8 \text { month assessment: } \\
\text { Analysed for objective 1: } n=14 \\
\text { Analysed for objective } 2: n=13 \\
12 \text { month assessment: } \\
\text { Analysed for objective } 1: n=12 \\
\text { Analysed for objective } 2: n=12\end{array}$ & & $\begin{array}{l}4 \text { month assessment: } \\
\text { Analysed for objective } 1 \text { and } 2: n=18 \\
\text { Incomplete data: } n=2 \\
8 \text { month assessment: } \\
\text { Analysed for objective } 1: n=14 \\
\text { Analysed for objective } 2: n=14 \\
12 \text { month assessment: } \\
\text { Analysed for objective } 1: n=13 \\
\text { Analysed for objective } 2: n=14\end{array}$ \\
\hline
\end{tabular}




\section{Analysis}

The research questions, analyses, and hypotheses of this trial were previously published [6].

\section{Psychological Markers}

Primary Outcome Measures

Fifty percent of the participants were abstinent from alcohol during the entire post-intervention period. TLFB scores were therefore dichotomized. On the basis of alcohol units per day, the sample was divided into participants who had not drunk at all and those who had one or more drinks per day. On the basis of \% days of heavy drinking, the sample was divided into participants who had been totally abstinent and those who had not been abstinent and accordingly for the \% days abstinent scale. Subsequently, a binary logistic regression model was run that included group as a predictor variable and time since detoxification as the covariate, separately for each TLFB variable.

\section{Secondary Outcome Measures}

ANCOVAs were conducted for the post-intervention, 4-month follow-up on the secondary outcome measures: HADS, BDI, OCDS, alcohol Stroop, and POMS. The type of treatment (NFT and TAU) was the between-group factor, and the baseline scores on the respective variable and participants' age, gender, and IQ were the covariates. For the DUQ, a binary logistic regression was performed because $>50 \%$ of the participants had the minimum value on each of the post-intervention assessments. The DUQ scores were dichotomized by splitting the sample into those participants who obtained the lowest possible score (a total score of 8) and participants who scored higher (total score $>8$ ). Next, a binary logistic regression model was run that included group as a predictor variable and time since detoxification as the covariate. As a precautionary measure, we determined whether participants had experienced increased craving in response to the alcohol stimuli. Pre- and post-session craving scores were then averaged across sessions and compared using confidence intervals.

\section{Sustained Effects}

Analysis for the TLFB was conducted as described for the 4-month time point. Differences between the TAU and NFT groups were assessed using ANCOVAs for each of the follow-up time points for the HADS, BDI, OCDS, alcohol Stroop, and POMS (8 and 12 months). Treatment (NFT and TAU) was the between-group factor, and base- line demographic variables (age, gender, and IQ) were included as covariates. For the DUQ, binary logistic regression was performed as described earlier.

\section{Preprocessing the Physiological Data}

For each scan, concurrent physiological data were processed using an in-house code to generate predictors of cardiac and respiratory noise [18-20]. Furthermore, the $\mathrm{P}_{\mathrm{ET}} \mathrm{CO}_{2}$ data were used as a predictor of fluctuations in arterial $\mathrm{CO}_{2}$ concentration, which is known to modulate the BOLD signal. Predictors were demeaned and convolved with their respective hemodynamic response function.

\section{Off-Line Analysis of MRI Data}

(f)MRI Data Preprocessing

Anatomical images were corrected for spatial intensity inhomogeneity and subsequently normalized into Montreal Neurological Institute (MNI) stereotactic space. Functional data were temporal high-pass filtered (GLM Fourier) with 2 cycles, 3D motion correction with trilinear estimation and sinc interpolation, linear trend removal, and slice scan time correction with cubic spline interpolation. For whole-brain analysis, 3D spatial smoothing with a Gaussian filter of $6.00 \mathrm{~mm}$ was applied. The fMRI data and regions of interest defined in $2 \mathrm{D}$ space were interpolated into $3 \mathrm{D}$ space using boundary-based registration and normalized into MNI space. All processing steps were performed using BrainVoyager 21.2 (Brain Innovation, Maastricht, the Netherlands).

\section{Whole-Brain Analysis}

A group random-effects GLM was calculated for each localizer task separately, including the types of image (alcohol/alternative goals or neutral), 6 motion parameters, and predictors of physiological noise as confounding predictors to estimate beta values. We employed a one-way, within-participants design with type of image (alcohol/ alternative goals or neutral) as the factor. Subsequently, alcohol/alternative goal-related images were contrasted with neutral images, and whole-brain maps were cluster corrected using Monte-Carlo simulations at a threshold of $p=0.001$. Additionally, the overall distribution of the neurofeedback target regions across participants was visualized to provide an overview on the main source of the neurofeedback information (see online suppl. material 1; see www.karger.com/doi/10.1159/000513448 for all online suppl. material). 


\section{ROI Activation}

Mean ROI activation (beta values) from the target areas in comparison to baseline was averaged across neurofeedback sessions within participants, separately for the 2 types of neurofeedback (alcohol and alternative goals, i.e., neurofeedback downregulation and upregulation) and task (neurofeedback or mirror run [passive viewing]). Effects of the neurofeedback were tested using paired $t$ tests, by comparing mean activation during neurofeedback with mean activation during mirror runs, separately for alcohol neurofeedback and alternative goal neurofeedback. For each individual, neurofeedback success was defined as lower (alcohol condition)/or higher (alternative goal condition) mean activation during neurofeedback runs compared to mirror runs.

Relationship between ROI Activation and Behavioral Outcome Measures (Exploratory)

To explore whether self-regulation performance during the neurofeedback intervention was related to changes in drinking behavior, overall self-regulation performance was compared between dichotomized groups of participants showing high/low outcomes on the TLFB\% of days abstinent and on the DUQ scores. TLFB amount of alcohol per day and \% days of heavy drinking were not included because they would have been redundant. Because all of the abstinent participants had not, of course, consumed any alcohol and had not engaged in any heavy drinking, the dichotomized post-intervention values were identical.

First, an overall marker of self-regulation performance was created by averaging self-regulation performance during alternative goal neurofeedback (mean beta [neurofeedback] - mean beta [mirror] of alternative goal sessions, weighted by $1 / 3$ ) with performance during alcohol neurofeedback (mean beta [neurofeedback] - mean beta [mirror] of alcohol sessions, weighted by $2 / 3$ ). Weighting reflected the performed number of NFT sessions for each condition, that is, $2 / 6$ or $4 / 6$ sessions, respectively. Second, to explore the relationship between drinking behavior (TLFB)/craving (DUQ) and overall neurofeedback performance, weighted average scores were descriptively explored by comparing mean performance between dichotomized groups (low/high values on the respective variable).

\section{Results}

\section{Psychological Markers}

Analysis on psychological markers could be performed for all measures and measurement time points; not all data points were available for all participants due to missing responses/measurement errors.

\section{Baseline Measures}

Baseline demographics were in line with expected demographics of the population: mean age $=45.8$ years, $\mathrm{SD}=9.9(\mathrm{NFT}$ mean $=45$ years $[\mathrm{SD}=9.9]$, control $=46$ years $[\mathrm{SD}=10.1])$, gender: $69 \%$ male, 36 males $(\mathrm{NFT}=$ $60 \%$ male [ 15 males], control $=78 \%$ male $[21$ males $]$ ). Time since detoxification at baseline was weighted over the whole sample: $1-3$ months: $n=25$ (48.0\%), 3-6 months: 27 (51.9\%); within groups: NFT 1-3 months: $n=12$ (48.0\%), 3-6 months: 13 (52.0\%); and control 1-3 months: $n=13$ (48.1), 3-6 months: 14 (51.9). Baseline scores for outcome measures showed no pronounced group differences (Table 1).

\section{Primary Outcome Measures}

Across all dichotomized markers of drinking behavior (TLFB scores) at the post-intervention (4-month) assessment, members of the neurofeedback group nominally showed lower alcohol consumption but with wide confidence intervals of the odds ratio that included 1 (Table 2 includes all patients who participated in the primary and secondary outcome assessments). These tendencies were also present in the undichotomized means for all of the measures, except for the amount of alcohol consumed per day (TLFB-amt/day), which showed a nominally higher value for the neurofeedback group (Tables 1A, 2). None of the group differences on the primary outcome measures would have been significant at the conventional level ( $p<0.05$, with Bonferroni correction for multiple outcome measures).

\section{Secondary Outcome Measures}

Members of the neurofeedback group nominally showed a lower probability of having an urge to drink, as indicated by the DUQ scores (Table 2). This tendency was also pronounced in the undichotomized means for the DUQ (Tables 1B, 2). ANCOVA of the OCDS also descriptively showed lower alcohol craving for the neurofeedback group (Table 2). Conversely, the alcohol Stroop interference was descriptively lower in the TAU group, and the affective measures also showed an inconsistent pattern (Table 2). None of the group differences on the secondary outcome measures would have been significant at the conventional level $(p<0.05$, with Bonferroni correction for multiple outcome measures). The additional safety measure tracking craving before and after the alcohol neurofeedback fRMI runs (via verbal assess- 


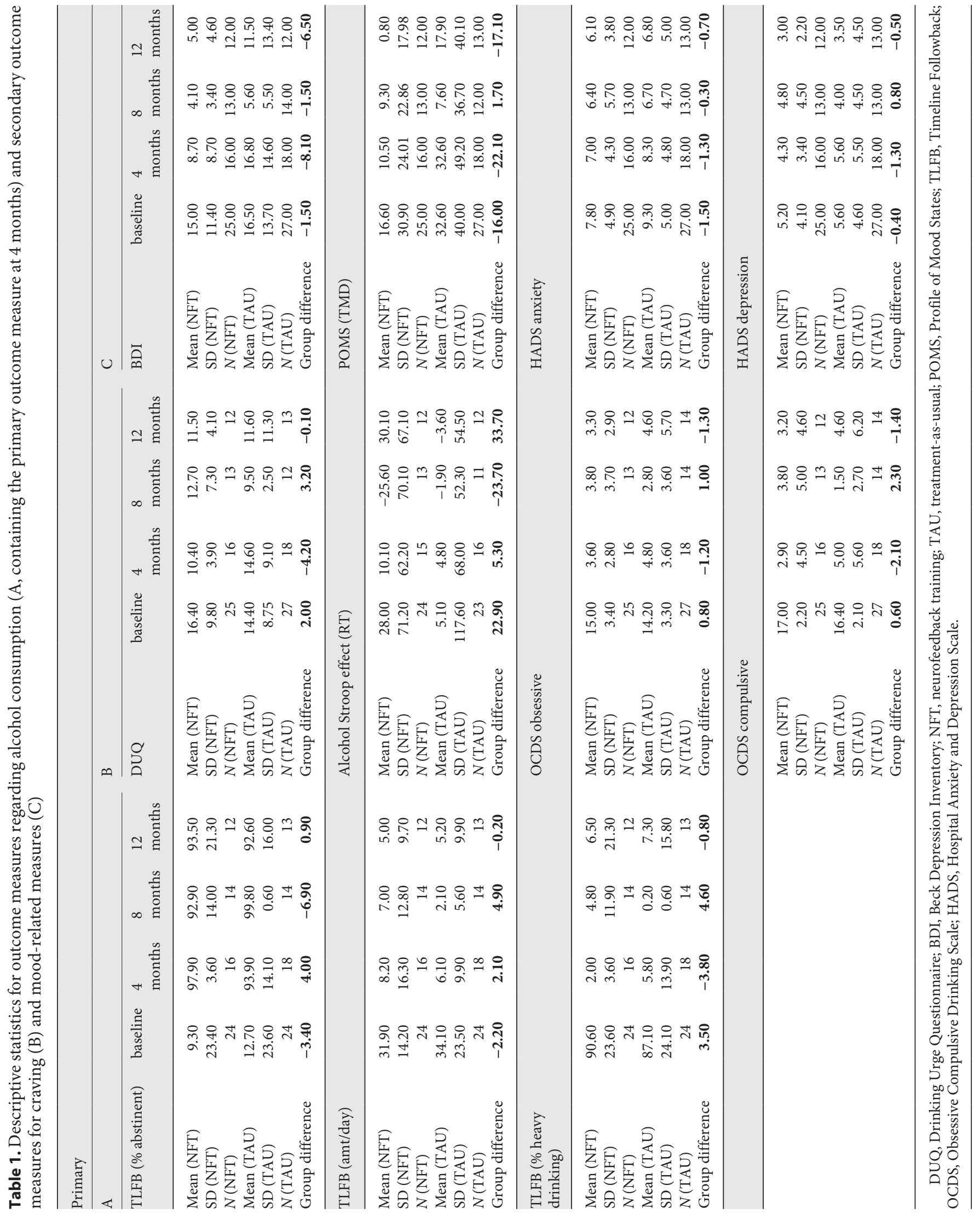


Table 2. Post-intervention group differences

\begin{tabular}{|c|c|c|c|c|}
\hline \multirow{2}{*}{$\begin{array}{l}\text { Primary outcome measures } \\
\text { regression analysis }\end{array}$} & \multirow[t]{2}{*}{$\operatorname{Exp}(B)$} & \multicolumn{2}{|c|}{$95 \%$ CI for $\operatorname{Exp}(B)$} & \multirow{2}{*}{$\begin{array}{l}\Delta \text { mean } \\
(\mathrm{NFT}-\mathrm{TAU})\end{array}$} \\
\hline & & lower & upper & \\
\hline$\%$ abstinent & 1.10 & 0.26 & 4.65 & \\
\hline Incl. detox & 1.11 & 0.26 & 4.70 & 4.00 \\
\hline Amount per day & 0.91 & 0.22 & 3.84 & \\
\hline Incl. detox & 0.90 & 0.21 & 3.83 & 2.10 \\
\hline$\%$ heavy drinking & 0.91 & 0.22 & 3.84 & \\
\hline Incl. detox & 0.90 & 0.21 & 3.83 & -3.80 \\
\hline \multirow{2}{*}{$\begin{array}{l}\text { Secondary outcome measures } \\
\text { regression analysis }\end{array}$} & \multirow[t]{2}{*}{$\operatorname{Exp}(B)$} & \multicolumn{2}{|c|}{$95 \%$ CI for $\operatorname{Exp}(B)$ or $F$} & \multirow{2}{*}{$\begin{array}{l}\Delta \text { mean } \\
(\text { NFT-TAU) }\end{array}$} \\
\hline & & lower & upper & \\
\hline DUQ & 0.29 & 0.07 & 1.20 & \\
\hline Incl. detox & 0.29 & 0.07 & 1.23 & -4.24 \\
\hline ANCOVA & $\mathrm{F}, \eta^{2} p$ & lower & upper & $\begin{array}{l}\triangle \text { EMM } \\
(\text { NFT-TAU) }\end{array}$ \\
\hline OCDS obsessive subscale & $F(1,27)=1.62, \eta^{2} p=0.06$ & -3.83 & 0.89 & -1.46 \\
\hline OCDS compulsive subscale & $F(1,27)=1.90, \eta^{2} p=0.07$ & -6.57 & 1.28 & -2.64 \\
\hline HADS depression & $F(1,27)=0.17, \eta^{2} p=0.01$ & -3.19 & 2.12 & -0.53 \\
\hline HADS anxiety & $F(1,27)=0.20, \eta^{2} p=0.01$ & -1.97 & 3.08 & 0.55 \\
\hline Alcohol Stroop & $F(1,22)=0.53, \eta^{2} p=0.02$ & -37.47 & 77.91 & 20.22 \\
\hline POMS (TMD) & $F(1,27)=0.21, \eta^{2} p=0.01$ & -18.11 & 28.39 & 5.13 \\
\hline BDI & $F(1,27)=2.07, \eta^{2} p=0.07$ & -12.29 & 2.16 & -5.06 \\
\hline
\end{tabular}

In regression analysis, probability is predicted for being a member of the neurofeedback group. TAU, treatment-as-usual; NFT, neurofeedback training; DUQ, Drinking Urge Questionnaire; OCDS, Obsessive Compulsive Drinking Scale; HADS, Hospital Anxiety and Depression Scale; POMS, Profile of Mood States; BDI, Beck Depression Inventory.

ment) did not indicate that craving was induced by the alcohol stimuli: mean (before) $=0.21(\mathrm{SD}=0.25)$; mean $($ after $)=0.22(\mathrm{SD}=0.29)$; mean difference (before minus after) $=-0.01 ; 95 \%$ CI for the difference: -0.11 to 0.92 . Considering the skewness of the differences (skewness = $-2.46, \mathrm{SE}=0.56$ ), a paired-sample sign test was additionally performed and confirmed that the craving scores did not increase from pre- to post-assessment (median before $=0.9, \mathrm{IQR}=0.42$; median after $=0.6, \mathrm{IQR}=0.38$; frequencies: negative differences [before $>$ after] $=4$; positive differences [before $<$ after] $=2$; ties $=10$ ).

Accordingly, comparison between the DUQ scores acquired before and after each neurofeedback session did not reveal a difference, and craving ratings at both time points were low: mean (before) $=11.48(\mathrm{SD}=4.76)$; mean $($ after $)=12.55(\mathrm{SD}=4.80)$; mean difference (before-after $)=-1.06$; $95 \%$ CI of the difference: -2.38 to 0.26 . Retention was $64 \%$ for the neurofeedback group (baseline: $n=25$, post-intervention: $n=16)$, and $67 \%$ for the TAU group (baseline: $n=27$, post-intervention: $n=18$ ).

Sustained Effects (8- and 12-Month Follow-Up)

Members of the TAU group compared to members of the NFT group showed a tendency toward less alcohol consumption (across all dichotomized markers of drinking behavior measured with the TLFB) 8 months after the start of the training, but not after 12 months, the confidence intervals included 0 (Table 3 ). This effect was also apparent in the group means of undichotomized variables (Table 1A). DUQ results for these follow-up time points are reported in Table 3 for the dichotomized values and in Table 1B for the undichotomized group means. ANCOVA results for the post-intervention time points are shown in Table 3 , and the descriptive values for the respective secondary outcome measures are shown in Table $1 \mathrm{~B}$ (craving-related measures) and Table 1C (mood-related measures). 
Table 3. Analysis of sustained effects

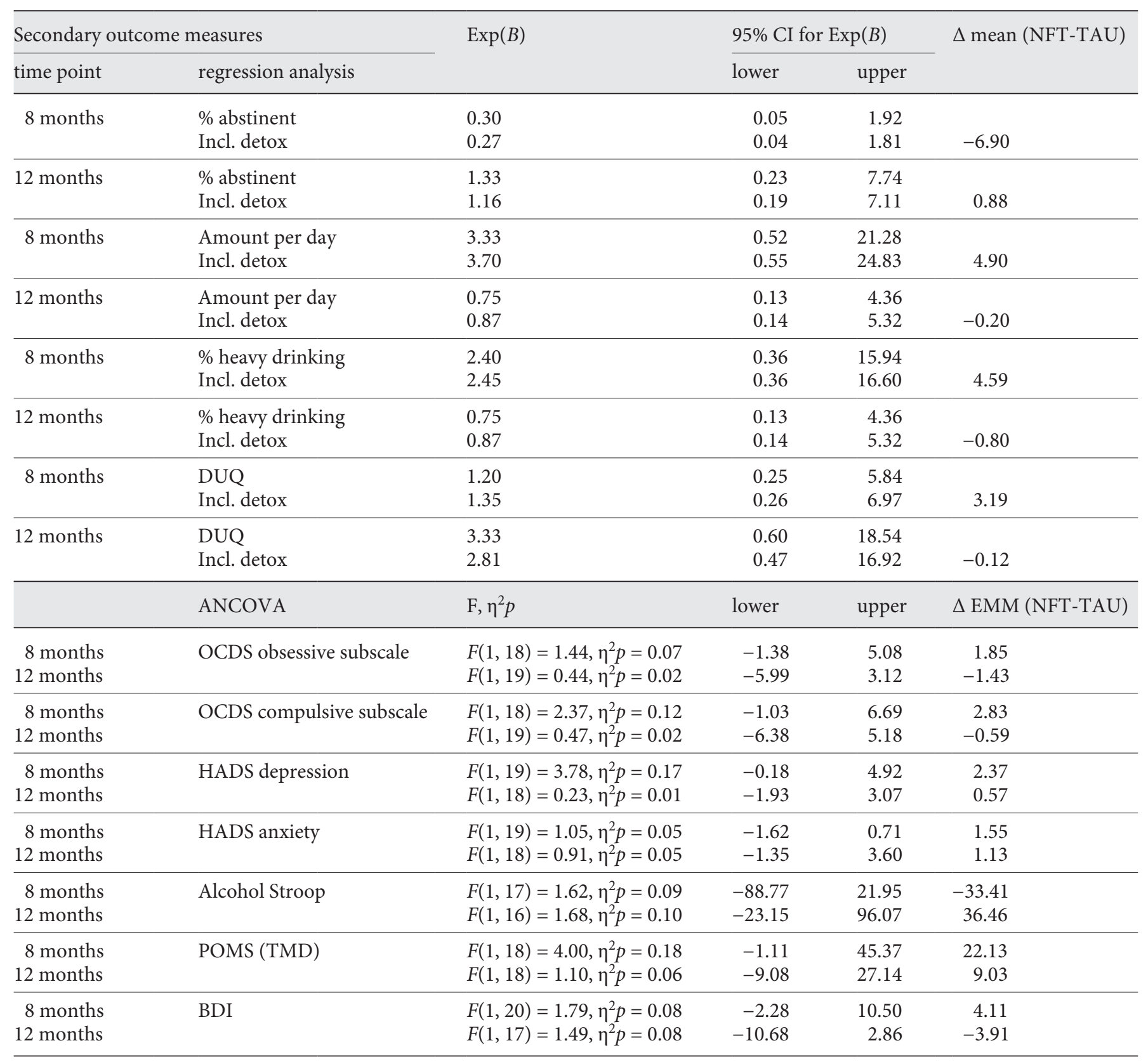

In regression analysis, probability is predicted for being a member of the neurofeedback group. TAU, treatment-as-usual; NFT, neurofeedback training; DUQ, Drinking Urge Questionnaire; OCDS, Obsessive Compulsive Drinking Scale; HADS, Hospital Anxiety and Depression Scale; POMS, Profile of Mood States; BDI, Beck Depression Inventory.

\section{fMRI Results}

Localizer Activation

Whole-brain analysis of the localizer data (Fig. 2) revealed pronounced activation for alcohol $>$ neutral in the reward system during the alcohol localizer, with signifi- cant activation in the right anterior cingulate cortex, the bilateral anterior insula, and bilateral striatum. There was also activation in the bilateral ventrolateral PFC (vLPFC), the right premotor cortex, and the posterior cingulate cortex. For the alternative goal localizer, activation was 


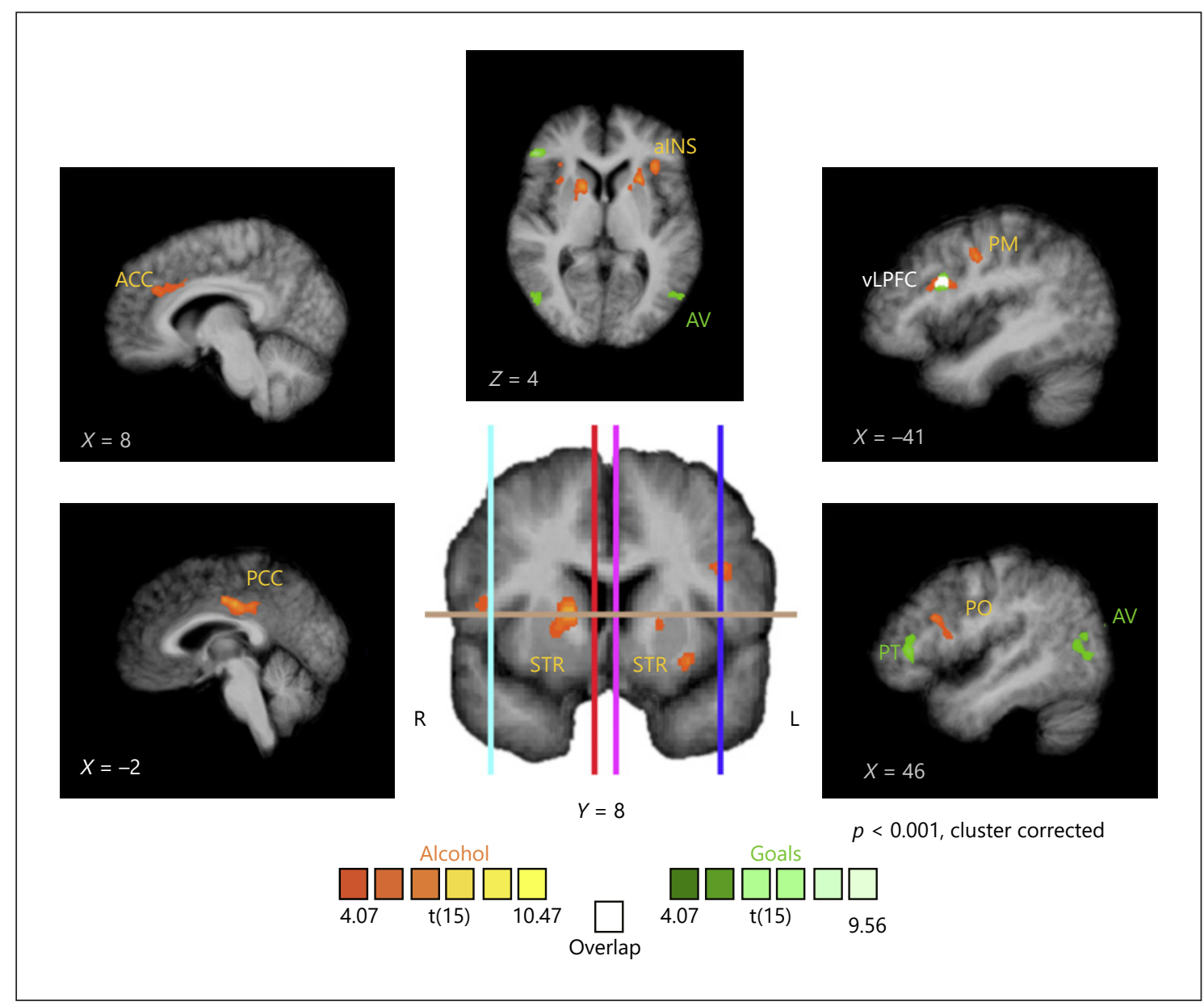

Fig. 2. Whole-brain activation (RFX results) across all sessions for the alcohol cue localizer (red/yellow) and alternative life goal localizer (green/mint). Activation maps are displayed on the average brain of all participants. aINS, anterior insula; ACC, anterior cingulate cortex; AV, visual associative areas; vLPFC, ventrolateral prefrontal cortex, PM, premotor cortex; PO, pars opercularis; PT, pars triangularis; STR, striatum; PCC, posterior cingulate cortex.

present only in cortical areas, that is, the bilateral vLPFC and the bilateral visual associative cortex. Activation clusters for both of the localizers overlapped in the left vLPFC.

\section{ROI Activation}

There was a trend for a difference between alcohol neurofeedback (mean betas across all sessions $=0.03$, $\mathrm{SD}=0.12)$ and alcohol mirror feedback (mean beta $=$ $0.09, \mathrm{SD}=0.16) ; t(15)=-1.93, p=0.07, \mathrm{EMM}$ difference $=-0.06$ (95\% CI -0.131 to 0.004) (Fig. 3a). Twelve of the 16 neurofeedback participants achieved lower mean activation during alcohol neurofeedback than during alcohol mirror feedback. There was a nominal, but nonsignificant, difference between alternative goal neu- rofeedback (mean $=0.04, \mathrm{SD}=0.12)$ and alternative goal mirror feedback (mean $=0.003, \mathrm{SD}=0.15) ; t(15)=0.84$, $p=0.4$, EMM difference $=-0.03(95 \% \mathrm{CI}-0.052$ to 0.120$)$ (Fig. 3b). Ten of the 16 neurofeedback participants achieved higher mean activation during alternative goal neurofeedback than during alternative goal mirror feedback.

Eight of the 16 participants were successful at self-regulation during both neurofeedback conditions (increased activation for alternative goals and decreased activation in response to alcohol stimuli during neurofeedback compared to mirror feedback). Six participants showed moderate performance, that is, successful regulation in one of the neurofeedback conditions (4 during alcohol 


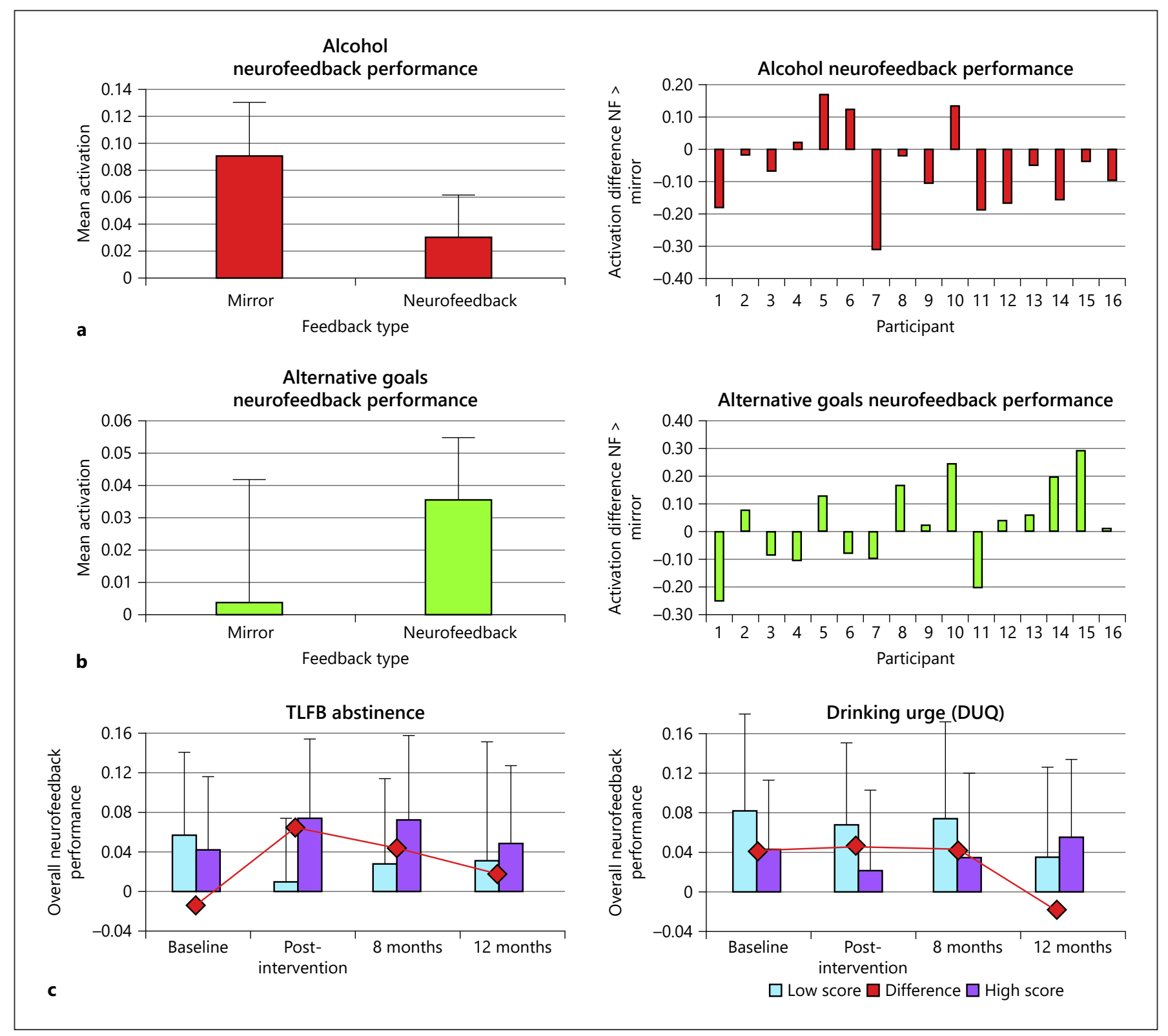

Fig. 3. Self-regulation performance and relationship to measures of craving/drinking behavior. a, b Group mean ROI activation (beta values, left) and self-regulation performance of individual participants (difference between ROI activation during neurofeedback and ROI activation during mirror feedback, right) for both training types. Error bars indicate standard errors. c Relationship between drinking behavior/craving and overall neurofeedback

performance. Mean neurofeedback performance separated between participants showing high values (turquoise) or low values (purple) of dichotomized TLFB/DUQ scores. Positive difference values (red line) indicate that high-performing participants showed desirable outcomes (lower > higher for DUQ; higher > lower for abstinence). Error terms indicate standard deviations. DUQ, Drinking Urge Questionnaire; TLFB, Timeline Followback.

neurofeedback and 2 during alternative goal neurofeedback). Two of the participants were not able to modulate the signal in either condition. (For distribution of the ROIs used as neurofeedback targets, see online suppl. material 1.) Individual differences in training success were

not driven by training with different scanner types (participants with successful alcohol NFT: GE $[n=3.5]: 2.5$, Siemens [ $n=12.5$ ]: 9.5 [ 1 participant performed half of the alcohol NFT training with each scanner]; positive goals NFT: GE [ $n=3]$ : 1 , Siemens [ $n=13]$ : 9 ). 
Relationship between ROI Activation and Behavioral Outcome Measures (Exploratory)

Overall, desirable outcomes (higher TLFB abstinence and lower urges to drink, DUQ) were associated with better neurofeedback performance (Fig. 3c). Across the 3 measures, this relationship was strongest at the post-intervention time point (highest difference in neurofeedback performance between those with low and those with high scores at month 4) and decreased during the followup period. Whereas drinking behavior at baseline (TLFB scores) appeared to be unrelated to neurofeedback performance, participants with lower drinking urges at baseline also showed overall better neurofeedback performance. Stratification of neurofeedback results into participants who remained abstinent until the post-intervention time point and participants who relapsed was in accordance with these findings for alcohol neurofeedback (mean difference neurofeedback $>$ mirror runs abstinent patients $(n=11)$ : mean $=-0.08, \mathrm{SD}=0.12$; non-abstinent patients $(n=5)$ : mean $=-0.03, \mathrm{SD}=0.15)$ and alternative goal neurofeedback (mean difference neurofeedback $>$ mirror runs abstinent patients $(n=11)$ : mean $=0.07, \mathrm{SD}=0.13$; non-abstinent patients $(n=5)$ : mean $=-0.04, \mathrm{SD}=0.21$ ).

\section{Adverse Events}

There was one serious adverse event (nonlethal overdose of paracetamol) in the TAU group, which was reported in accordance with the standard procedures of the South East Wales Trials Unit and judged to be unrelated to the intervention.

\section{Discussion}

Feasibility of fMRI-NFT as a Treatment for Alcohol Dependence

The patient retention and MRI data show that fMRIbased NFT for patients with alcohol dependence is feasible. A retention rate of $64 \%$ in the intervention group for an intensive intervention running over a 4 -week period is acceptable for patients with a substance use disorder. Analysis of neurofeedback performance showed that participants were able to use the feedback as instructed. Furthermore, indices of craving acquired during the neurofeedback sessions revealed low overall craving in patients during the neurofeedback sessions and no significant increases from before to after the neurofeedback sessions, suggesting that the craving induction presented little risk. The relationship between overall task perfor- mance and less alcohol consumption and craving suggest that during the NFT, clinically relevant processes were modulated. However, given the lack of differences in clinical outcomes between the NFT + TAU group and the TAU only group, we cannot infer that neurofeedback was specifically instrumental in maintaining the high abstinence rates observed.

High levels of abstinence post-intervention in both groups (11/16 in NFT + TAU group, 12/18 in TAU group) and low alcohol consumption in the patients who did drink diminished the possibility of detecting group differences. The abstinence rate in the TAU group was higher than expected from the literature on the long-term effects of inpatient treatment. Relapse rates have been as high as $70-80 \%$ by the end of the first year, and they have typically been in the range of $40-60 \%$ within the first few months after treatment [21]. In the present study, therefore, patients with a high probability of relapse had already relapsed when recruitment began, and they were not included. Overall, 2 conclusions can therefore be drawn from this trial: the NFT was itself feasible, but the target population seemed too resilient to benefit from the neurofeedback intervention.

\section{Neural Effects}

The fMRI results provide further support for the feasibility of NFT to modulate the neural mechanisms of interest. Activation during the localizer procedure indicated that exposure to the visual stimuli engaged the functionally relevant brain systems (Fig. 2), with strong involvement of the reward system and cognitive control areas in the lateral prefrontal cortex which was accordingly reflected in the individualized target regions (online suppl. Fig. 1,2). Conceptually, increased cue reactivity of the reward/salience system in people with alcohol dependence [22-26] is an attractive target for interventions that aim to promote abstinence and reduce craving (see, e.g., $[27,28])$. Considering the predictive association between neural cue reactivity and alcohol consumption [29], techniques aiming to attenuate this hyperactivity thus have potential utility as a treatment for alcohol use disorders (e.g., [26]). The feasibility of the attenuation through NFT, which was demonstrated here for the first time, supports the utility of adding motivational neurofeedback [30] to the toolkit of interventions designed for substance use disorders.

\section{Implications for Future Studies}

A limitation of the present design was that participant blinding was not possible. Due to the general prob- 
lem of identifying an appropriate control condition for neurofeedback [31], controlling for confounds associated with unblinded designs would require the inclusion of several study arms. Considering the lack of research on neurofeedback for alcohol use disorders, it is therefore more economical to first determine whether its clinical effects relative to existing treatments justify an investment in larger studies. Once a design (either alone or in addition to standard care) has been shown to be (potentially) superior to standard care, it should be tested against active control interventions. Future multiarmed studies could include a sham condition in a blinded design, in which patients receive predetermined visual stimulation matched with regard to the positive feedback provided during neurofeedback, as well as self-regulation training without feedback, to control for generic effects of self-regulation and placebo effects $[31,32]$. Considering the results of this study, subsequent research should also focus on different patient target groups for which neurofeedback could be more effective. One possibility would be to conduct the training immediately after detoxification training, in order to prevent relapse in a group that is at the highest risk of relapse. Another relevant question is whether early-stage problem drinkers could benefit from a neurofeedback intervention. Early-stage problem drinkers are far more numerous than dependent drinkers, and a neurofeedback intervention could help them moderate their drinking before it escalates into dependent drinking. Additionally, as more general individual differences like psychological traits could also influence how patients react to the neurofeedback setting, assessments of such traits could help individualize subsequent clinical trials [33]. In line with this notion, higher alcohol craving at baseline tended to be associated with lower neurofeedback performance, which appeared not to be the case for drinking behavior itself. Although these findings are only descriptive at this point, they support that individual differences in craving could indicate how patients will respond to neurofeedback interventions.

Besides improving the selectivity of neurofeedback interventions with regard to the target population, the training procedure itself should be optimized. For example, additional self-regulation training outside of the scanner could be a cost-effective solution. Increasing the NFT time itself could also facilitate training effects but will require a cost-benefit evaluation. Additionally, changes in the neurofeedback procedure itself could improve training efficacy, but optimizing this component will likely require meta-analytic comparisons be- tween several trials as the search space for potential adaptions is extensive (e.g., neurofeedback could be built from mean activation of different regions, connectivity between different regions, or multivariate activation pattern of different regions). Overall, further studies are required to determine clinical efficacy and to understand the influence of placebo effects, individual differences, and the generic effects of self-regulation trainings.

\section{Acknowledgements}

We are very grateful to the staff of the Health and Care Research Wales Clinical Research Centre and the National Centre for Mental Health (NCMH), particularly Rebecca Robinson, Sophie Bishop, and Andrew Bethell, for their help with patient recruitment, and to all the local clinics and rehabilitation centers, charities, and patient support groups for their support in recruiting participants. We are also very grateful to Jacqui Smith and Rachael Adams for conducting the blinded assessments.

\section{Statement of Ethics}

This study was approved by the Wales Research Ethics Committee 1 (Ref: 14/WA/1172), the Cardiff School of Psychology Ethics Committee, and the Cwm Taf, Cardiff and Vale University, and Aneurin Bevan University Health Boards. All participants gave written informed consent to participate in the study in accordance with the Declaration of Helsinki. This trial was registered with Clinicaltrials.gov (NCT02486900) in June 2015 before the first participant was recruited. This study was completed in August 2018 after acquisition of the final follow-up assessments.

\section{Conflict of Interest Statement}

M.L. is an employee at and R.G. is owner of Brain Innovation (B.V.), the company that produces software used for the neurofeedback setup. The other authors have no conflicts of interest to declare.

\section{Funding Sources}

This study was conducted by the BRAINTRAIN consortium (partners Cardiff University and Brain Innovation), supported by the European Commission, under the Health Cooperation Work Programme of the 7th Framework Programme, under the Grant Agreement Nr. 602186. The Centre for Trials Research is funded by Health and Care Research Wales and Cancer Research UK. 


\section{Author Contributions}

Conception and design of the work: W.M.C., K.H., R.S., R.P., R.McN., G.W., N.I., R.G., and D.E.J.L. Acquisition, analysis, or interpretation of data: all authors. Drafting the work or revising it critically for important intellectual content and approving the final version to be published: all authors. Agreed to be accountable for all aspects of the work in ensuring that questions related to the accuracy or integrity of any part of the work are appropriately investigated and resolved: all authors.

\section{References}

1 Canterberry M, Hanlon CA, Hartwell KJ, Li $\mathrm{X}$, Owens M, LeMatty T, et al. Sustained reduction of nicotine craving with real-time neurofeedback: exploring the role of severity of dependence. Nicotine Tob Res. 2013; 15(12):2120-4

2 Hanlon CA, Hartwell KJ, Canterberry M, Li $\mathrm{X}$, Owens M, LeMatty T, et al. Reduction of cue-induced craving through realtime neurofeedback in nicotine users: the role of region of interest selection and multiple visits. Psychiatry Res. 2013;213(1):79-81.

3 Li X, Hartwell KJ, Borckardt J, Prisciandaro JJ, Saladin ME, Morgan PS, et al. Volitional reduction of anterior cingulate cortex activity produces decreased cue craving in smoking cessation: a preliminary real-time fMRI study. Addict Biol. 2013;18(4):739-48.

4 Karch S, Keeser D, Hümmer S, Paolini M, Kirsch V, Karali T, et al. Modulation of craving related brain responses using real-time fMRI in patients with alcohol use disorder. PLoS One. 2015;10(7):e0133034.

5 Kirsch M, Gruber I, Ruf M, Kiefer F, Kirsch P. Real-time functional magnetic resonance imaging neurofeedback can reduce striatal cuereactivity to alcohol stimuli. Addict Biol. 2016;21(4):982-92.

6 Cox WM, Subramanian L, Linden DE, Lührs M, McNamara R, Playle R, et al. Neurofeedback training for alcohol dependence versus treatment as usual: study protocol for a randomized controlled trial. Trials. 2016;17(1): 480.

7 Cox WM, Klinger E. A motivational model of alcohol use. J Abnorm Psychol. 1988;97(2): 168.

8 Cox WM, Klinger E. A motivational model of alcohol use: determinants of use and change. In: Cox WM, Klinger E, editors. Handbook of motivational counseling: goal-based approaches to assessment and intervention with addiction and other problems. Hoboken, NJ: Wiley Blackwell; 2011. p. 131-58.

9 Cox WM, Klinger E, Fadardi JS. The motivational basis of cognitive determinants of addictive behaviors. Addict Behav. 2015;44:1622.
10 Ihssen N, Cox WM, Wiggett A, Fadardi JS, Linden DE. Differentiating heavy from light drinkers by neural responses to visual alcohol cues and other motivational stimuli. Cereb Cortex. 2010;21(6):1408-15.

11 Sobell LC, Sobell MB. Timeline follow-back: a technique for assessing self-reported alcohol consumption. 1992.

12 Bohn MJ, Krahn DD, Staehler BA. Development and initial validation of a measure of drinking urges in abstinent alcoholics. Alcohol Clin Exp Res. 1995;19(3):600-6.

13 Roberts JS, Anton RF, Latham PK, Moak DH. Factor structure and predictive validity of the obsessive compulsive drinking scale. Alcohol Clin Exp Res. 1999;23(9):1484-91.

14 Cox WM, Fadardi JS, Pothos EM. The addiction-stroop test: theoretical considerations and procedural recommendations. Psychol Bull. 2006;132(3):443.

15 Heuchert J, McNair D. Profile of mood states. 2012.

16 Zigmond AS, Snaith RP. The hospital anxiety and depression scale. Acta Psychiatr Scand. 1983;67(6):361-70.

17 Beck AT, Steer RA, Brown GK. Beck depression inventory-II. San Antonio. 1996;78(2): 490-8.

18 Glover GH, Li TQ, Ress D. Image-based method for retrospective correction of physiological motion effects in fMRI: RETROICOR. Magn Reson Med. 2000;44(1):162-7.

19 Birn RM, Smith MA, Jones TB, Bandettini $\mathrm{PA}$. The respiration response function: the temporal dynamics of fMRI signal fluctuations related to changes in respiration. Neuroimage. 2008;40(2):644-54.

20 Chang C, Cunningham JP, Glover GH. Influence of heart rate on the BOLD signal: the cardiac response function. Neuroimage. 2009; 44(3):857-69.

21 Dawson DA, Goldstein RB, Grant BF. Rates and correlates of relapse among individuals in remission from DSM-IV alcohol dependence: a 3-year follow-up. Alcohol Clin Exp Res. 2007;31(12):2036-45.

22 Grüsser SM, Wrase J, Klein S, Hermann D, Smolka MN, Ruf M, et al. Cue-induced activation of the striatum and medial prefrontal cortex is associated with subsequent relapse in abstinent alcoholics. Psychopharmacology. 2004;175(3):296-302.
23 Heinz A, Beck A, Grüsser SM, Grace AA, Wrase J. Identifying the neural circuitry of alcohol craving and relapse vulnerability. Addict Biol. 2009;14(1):108-18.

24 Klinger E, Cox WM. Motivation and the goal theory of current concerns. In: Handbook of motivational counseling. 2011. Vol. 3; p. 47.

25 Schacht JP, Anton RF, Myrick H. Functional neuroimaging studies of alcohol cue reactivity: a quantitative meta-analysis and systematic review. Addict Biol. 2013;18(1):121-33.

26 Wiers CE, Stelzel C, Gladwin TE, Park SQ, Pawelczack S, Gawron CK, et al. Effects of cognitive bias modification training on neural alcohol cue reactivity in alcohol dependence. Am J Psychiatry. 2015;172(4):335-43.

27 Robinson TE, Berridge KC. The neural basis of drug craving: an incentive-sensitization theory of addiction. Brain Res Brain Res Rev. 1993;18(3):247-91.

28 Berridge KC, Robinson TE. Liking, wanting, and the incentive-sensitization theory of addiction. Am Psychol. 2016;71(8):670.

29 Courtney KE, Schacht JP, Hutchison K, Roche DJ, Ray LA. Neural substrates of cue reactivity: association with treatment outcomes and relapse. Addict Biol. 2016;21(1): $3-22$.

30 Sokunbi MO, Linden DE, Habes I, Johnston $\mathrm{S}$, Ihssen N. Real-time fMRI brain-computer interface: development of a "motivational feedback" subsystem for the regulation of visual cue reactivity. Front Behav Neurosci. $2014 ; 8: 392$

31 Sorger B, Scharnowski F, Linden DEJ, Hampson M, Young KD. Control freaks: towards optimal selection of control conditions for fMRI neurofeedback studies. Neuroimage. 2019;186:256-65.

32 Young KD, Zotev V, Phillips R, Misaki M, Yuan H, Drevets WC, et al. Real-time FMRI neurofeedback training of amygdala activity in patients with major depressive disorder. PLoS One. 2014;9(2):e88785.

33 Fede SJ, Dean SF, Manuweera T, Momenan R. A guide to literature informed decisions in the design of real time fMRI neurofeedback studies: a systematic review. Front Hum Neurosci. 2020;14:60. 